\title{
Search for Dark Matter by GENIUS-TF and GENIUS
}

\author{
H.V. Klapdor-Kleingrothaus ${ }^{\text {a* }}$ \\ ${ }^{a}$ Max-Planck-Institut für Kernphysik, P.O. Box 1039 80, \\ D-69029 Heidelberg, GERMANY
}

The new project GENIUS will cover a wide range of the parameter space of predictions of SUSY for neutralinos as cold dark matter. Together with DAMA it will be the only experiment which can probe the seasonal modulation signal. Concerning hot dark matter GENIUS will be able to fix the (effective) neutrino mass with high accuracy. A GENIUS Test Facility has just been funded and will come into operation by end of 2002 .

\section{GENERAL SITUATION}

Dark matter is at present one of the most exciting fields of particle physics and cosmology. Recent investigations of the CMB (MAXIMA, BOOMERANG, DASI) together with large scale structure results fix the non-baryonic dark matter contribution to the mass of the Universe to $25 \%$, of which still up to $38 \%$ could be hot (neutrinos). With the early nucleosynthesis constraint of $\Omega=0.04$, the overwhelming contribution would consist of dark energy.

Terrestrial direct dark matter search experiments just now are starting to enter into the range of sensitivity required, it we consider the neutralinos to be the candidate for cold dark matter. The situation is similar in the hot Dark Matter Search. Here double beta decay is the most sensitive means to look for an absolute neutrino mass scale.

Direct search for WIMPs can be done

(a) by looking for the recoil nuclei in WIMPnucleus elastic scattering. The signal could be ionisation, phonons or light produced by the recoiling nucleus. The typical recoil energy is a few $100 \mathrm{eV} / \mathrm{GeV}$ WIMP mass.

(b) by looking for the modulation of the WIMP signal resulting from the seasonal variation of the earth's velocity against the WIMP 'wind'.

The expectation for neutralino elastic scattering cross sections and masses have been exten-

\footnotetext{
* Spokesman of HEIDELBERG-MOSCOW and GENIUS Collaboratiops E-mail: klapdor@gustav_mpi-hd.mpg, Home-page: http://www.mpi-hd.mpg.de.non_acc/
}

sively analysed in many variants of SUSY models.

Fig. 1 represents the present situation. The SUSY predictions are from the MSUGRA model [2] and the MSSM with relaxed unification conditions [1]. Fig. 2 shows the result of a study 'at Post-LEP Benchmark points' based again on the MSUGRA 3. Present experiments only just touch the border of the area predicted by the MSSM. The experimental DAMA evidence for dark matter lies in an area, in which MSUGRA models do not expect dark matter. They would require Beyond-GUT physics in this frame [5].

Summarizing the present experimental status, present experiments and also future projects (see [6]) can be categorized in two classes:

1. Sensitivity (or sensitivity goal) 'just for' confirmation of DAMA.

2. Sensitivity to enter deeply into the range of SUSY predictions.

Only very few experiments may become candidates for category 2 in a foreseeable future (see Figs. 1,2, and as far as at present visible, of those only GENIUS will have the chance to search for modulation, i.e. to check, like DAMA, positive evidence for a dark matter signal.

The cryogenic experiments CDMS, CRESST and Edelweiss operate at present with $600 \mathrm{~g}$ of detectors and less, after a decade of development. Expansion to several tens of $\mathrm{kg}$ or better $100 \mathrm{~kg}$ as required for modulation search, is still a very large step. CDMS has collected only $10.6 \mathrm{~kg} \mathrm{~d}$ of data over this time (in 1999, since then not working), Edelweiss only $4.53 \mathrm{~kg} \mathrm{~d}$.

The superheated droplet detectors PI- 


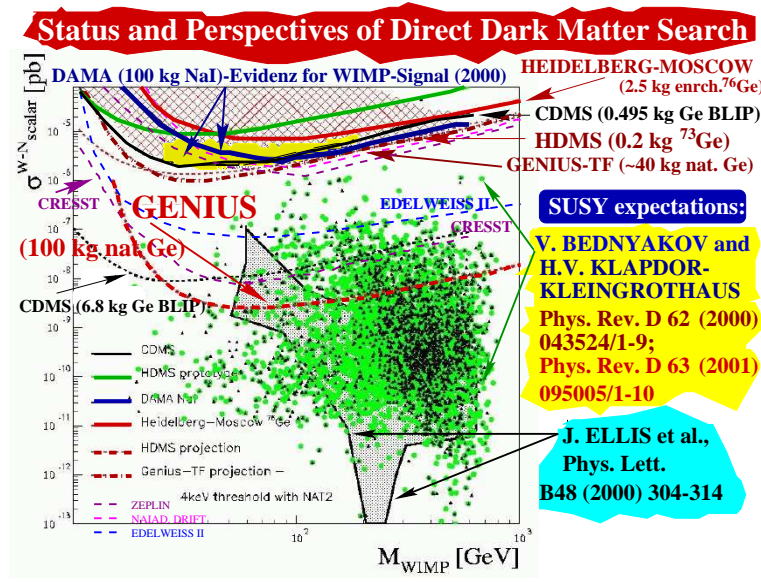

Figure 1. WIMP-nucleon cross section limits in $\mathrm{pb}$ for scalar interactions as function of the WIMP mass in GeV. Shown are contour lines of present experimental limits (solid lines) and of projected experiments (dashed lines). Also shown is the region of evidence published by DAMA. The theoretical expectations are shown for the MSSM by two scatter plots, - for accelerating and for non-accelerating Universe (from [1]) and for MSUGRA by the grey region (from [21). Only GENIUS will be able to probe the shown range also by the signature from seasonal modulations.

CASSO/SIMPLE are working at present on a scale of 15 and $50 \mathrm{~g}$ detectors, respectively.

ZEPLIN and the very ambiguous and nice project DRIFT are in the stage of prototype construction.

The situation is different for GENIUS, which is based on conventional techniques.

\section{GENIUS AND COLD DARK MAT- TER SEARCH}

If classifying the SUGRA models into more $\mathrm{g}_{\mu}$-2-friendly (I,L,B,G,C,J) and less $\mathrm{g}_{\mu}$-2-friendly models, according to [3], the former ones have good prospects to be detectable by LHC and/or a $1 \mathrm{TeV}$ collider. GENIUS could check not only the larger part of these ones, but in addition two of the less $\mathrm{g}_{\mu}$-2-friendly models ( $\mathrm{E}$ and $\mathrm{F}$ ), which will be difficult to be probed by future colliders (see Fig. 2).

This demonstrates nicely the complementarity of collider and underground research.

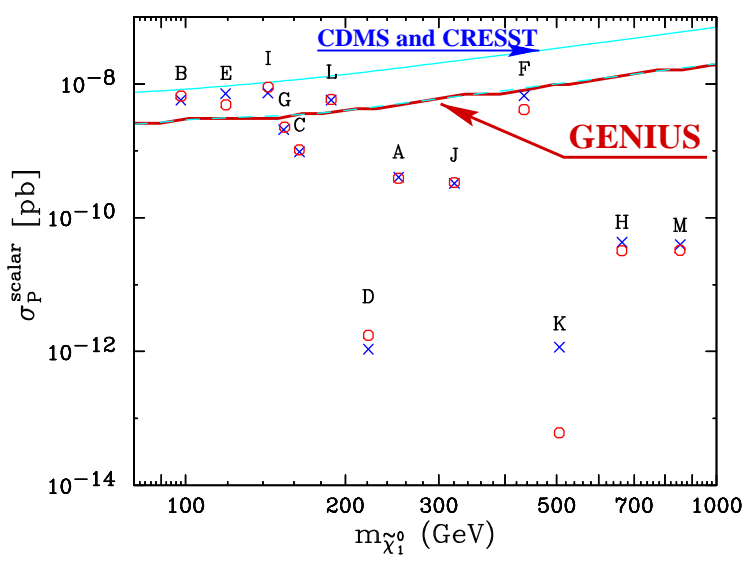

Figure 2. WIMP- proton elastic scattering cross sections according to various MSUGRA models (see text). From [3].

GENIUS would already in a first step, with $100 \mathrm{~kg}$ of natural Ge detectors, cover a significant part of the SUSY parameter space for prediction of neutralinos as cold dark matter (Figs. 1ㄹㄹ). For this purpose the background in the energy range $<100 \mathrm{keV}$ has to be reduced to $10^{-2}$ (events/ kg y keV). At this level solar neutrinos as source of background are still negligible. Of particular importance is to shield the detectors during production (and transport) to keep the background from spallation by cosmic rays sufficiently low (for details see [16].

The sensitivity of GENIUS for Dark Matter corresponds to that obtainable with a $1 \mathrm{~km}^{3}$ AMANDA detector for indirect detection (neutrinos from annihilation of neutralinos captured at the Sun) (see [17]). Interestingly both experiments would probe different neutralino compositions: GENIUS mainly gaugino-dominated neutralinos, AMANDA mainly neutralinos with comparable gaugino and Higgsino components (see Fig. 38 in 117). 


\section{GENIUS AND HOT DARK MATTER SEARCH}

Neutrinos could still play an important role as hot dark matter in the Universe. Recent results from CMB and LSS measurements still allow for a sum of neutrino masses of $\sum m_{i}<4.4 . \mathrm{eV}$. Under the assumption that neutrinos are degenerate in mass, the common mass eigenvalue is, from $0 \nu \beta \beta$ decay, $<1.4 \mathrm{eV}$ (90\% c.l.) if the LMA MSW solution is realized in nature 18. New approaches and considerably enlarged experiments, will be required in future to fix the neutrino mass with higher accuracy. GENIUS is probably the most straightforward and promising of them. As discussed 17 15,, 4 it could probe with 1 ton of enriched ${ }^{76} \mathrm{Ge}$ a scale of the effective neutrino mass down to $0.02 \mathrm{eV}$. This means that with $100 \mathrm{~kg}$ of enriched ${ }^{76} \mathrm{Ge}$, a scale down to $0.04 \mathrm{eV}$ could be investigated.

\section{GENIUS-TF}

As a first step of GENIUS, a small test facility, GENIUS-TF, is at present under installation in the Gran Sasso Underground Laboratory 19. With about $40 \mathrm{~kg}$ of natural Ge detectors operated in liquid nitrogen, GENIUS-TF could test the DAMA seasonal modulation signature for dark matter. Up to summer 2001, already six 2.5 $\mathrm{kg}$ Germanium detectors with an extreme lowlevel threshold of $\sim 500 \mathrm{eV}$ have been produced.

\section{CONCLUSION}

Terrestrial dark matter experiments searching for cold dark matter only now are starting to enter into the range of SUSY predictions. GENIUS and GENIUS-TF could play a decisive role here, as also in fixing the contribution of neutrinos to hot dark matter.

\section{REFERENCES}

1. V.A. Bednyakov and H.V. Klapdor-Kleingrothaus, Phys. Rev. D 62 (2000) 043524/1, and Phys. Rev. D 63 (2001) 095005.

2. J. Ellis et al. Phys. Lett. B 481 (2000) 304 and Phys. Rev. D 63 (2000) 065016.

3. J.Ellis etal. hep-ph/0111294,hep-ph/0202110.
4. H.V. Klapdor-Kleingrothaus, "60 Years of Double Beta Decay", World Scientific, Singapore (2001) $1253 \mathrm{p}$.

5. R. Arnowitt, private communication, 2001

6. H.V. Klapdor-Kleingrothaus, in Proc. of LP01, World Scientific, Singapore (2002) and of NANP01 Part. and Nucl. Lett. (2002).

7. H.V. Klapdor-Kleingrothaus, in Progr. in Part. and Nucl. Phys. 48 (2002).

8. H.V. Klapdor-Kleingrothaus in Proc. of BEYOND'97, Castle Ringberg, Germany, 814.06.1997, edited by H.V. Klapdor-Kleingrothaus and H. Päs, IOP, Bristol (1998) 485.

9. H.V. Klapdor-Kleingrothaus et al. MPIReport MPI-H-V26-1999 and hepph/9910205 and in Proc. of BEYOND'99, Castle Ringberg, Germany, 6-12.06.1999, ed. by H.V. Klapdor-Kleingrothaus and I.V. Krivosheina, IOP, Bristol (2000) 915.

10. H.V. Klapdor-Kleingrothaus, in Proc. of NEUTRINO'98, (eds) Y. Suzuki et al. Nucl. Phys. Proc. Suppl. 77 (1999) 357.

11. H.V. Klapdor-Kleingrothaus, in Proc. of WEIN'98, P. Herczeg, C.M. Hoffman and H.V. Klapdor-Kleingrothaus (Eds.), World Scientific, Singapore (1999) 275.

12. H.V. Klapdor-Kleingrothaus, in Proc. of NOW2000, ed. G. Fogli, Nucl. Phys. Proc. Suppl. B 100 (2001) 309 and 350.

13. H.V. Klapdor-Kleingrothaus, J. Hellmig and M. Hirsch, J. Phys. G 24 (1998) 483.

14. H.V. Klapdor-Kleingrothaus, Int. J. Mod. Phys. A 13 (1998) 3953.

15. H.V. Klapdor-Kleingrothaus, Springer Tracts in Mod. Phys., 163 (2000) 69.

16. H.V. Klapdor-Kleingrothaus, in Proc. LowNu2 and NOON2000, ed: Y. Suzuki, World Scientific, Singapore (2001).

17. J. Edsjö, h.p.: http://www.physto.se/edsjo/.

18. H.V. Klapdor-Kleingrothaus et al. H. Päs and A.Yu. Smirnov, Phys. Rev. D 63 (2001) 095005; hep-ph/0103076.

19. H.V. Klapdor-Kleingrothaus et al., hepex/0012022, Subm. for Publ. (2002). 